\title{
Téoros
}

Revue de recherche en tourisme

\section{Le stage en milieu professionnel dans la formation en tourisme}

\section{Jean-Marc Descôteaux}

Volume 11, numéro 1, mars 1992

La formation en tourisme : à la croisée des chemins

URI : https://id.erudit.org/iderudit/1078944ar

DOI : https://doi.org/10.7202/1078944ar

Aller au sommaire du numéro

Éditeur(s)

Université du Québec à Montréal

ISSN

0712-8657 (imprimé)

1923-2705 (numérique)

Découvrir la revue

Citer cet article

Descôteaux, J.-M. (1992). Le stage en milieu professionnel dans la formation en tourisme. Téoros, 11(1), 17-23. https://doi.org/10.7202/1078944ar d'utilisation que vous pouvez consulter en ligne.

https://apropos.erudit.org/fr/usagers/politique-dutilisation/ 


\section{Le stage en milieu professionnel dans la formation en tourisme}

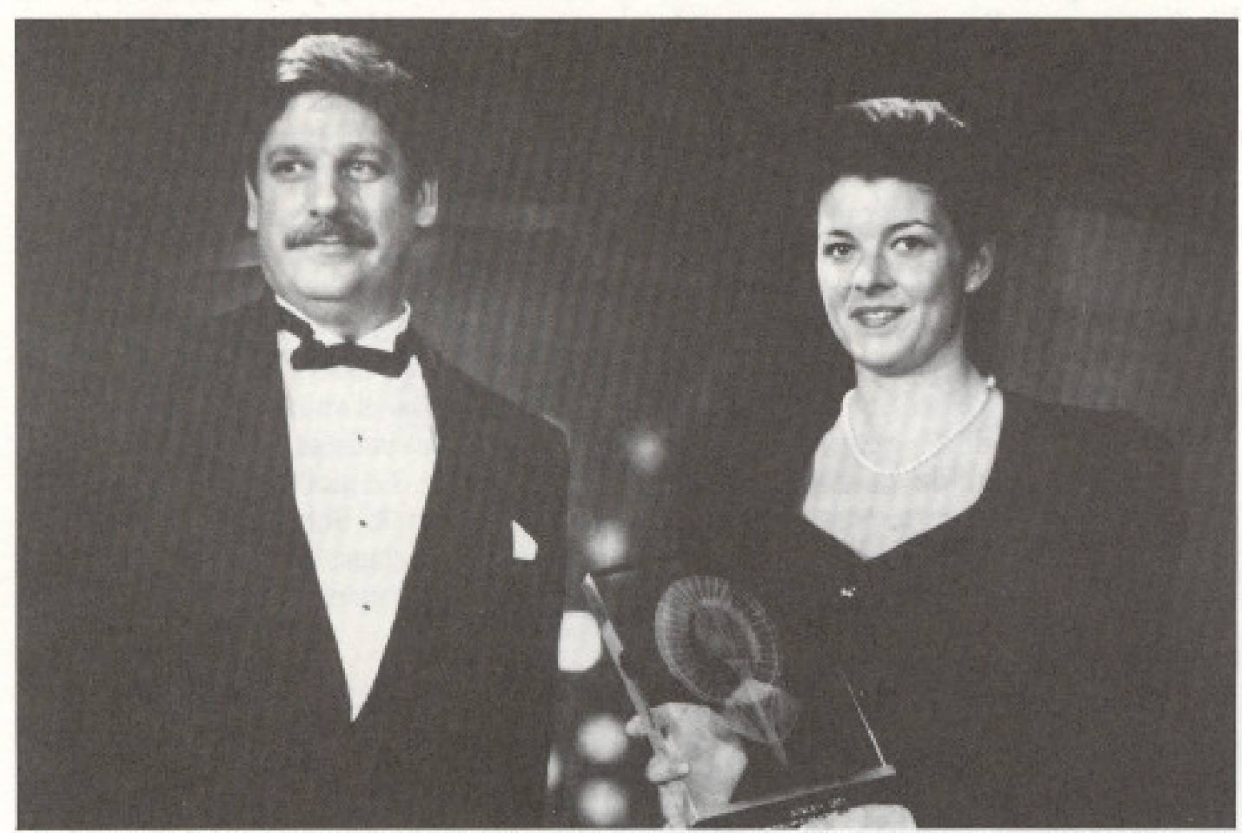

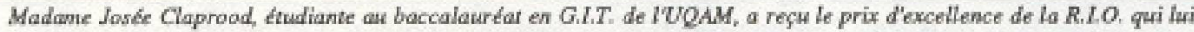

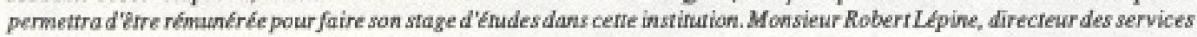
fouristipase de la R.I.O. Lui a remis ee prix.

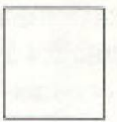

Quin'apas déjàentenduoumême constaté que les réalités et les enseignements reçus en milieu universitaire sontparfois, voiremême souvent,éloignés de la vie réelle en milieu de travail? Voilà pourquoi nous tenterons ici d'analyser la problématique des stages en tourisme et d'en déceler l'importance stratégique dans le cheminement professionnel et la formation des étudiants. Cette réflexion s'inscrit dans une large démarche, perceptible dans l'ensemble des milieux universitaires. Aux quatre coins du Québec, les différentes institutions réorientent leurs programmes pour faire face aux nouveaux defis du marché et de la société ${ }^{[a]}$.

\section{Qu'est-ce qu'un stage?}

L'exemple classique demeure àce niveau les programmes Coop de l'Université de Sherbrooke. Cette formule, introduite en ingénierie et au MBA en 1966, représentait

Monsieur.Jean-Marc Descóteaux est chargéde cours

au Département d'otudes urbaines et touristiques a

I'Université du Québec à Montréal. alors la première initiative québécoise vers une révision de l'enseignement, purement et strictement magistral, dans les universités. Quelque 25 ans plus tard et étendue à la majorité des programmes de l'Université de Sherbrooke, force est de constater que ula formule permet (...) aux étudiants d' aoquérir une solide formation théorique de même qu"une expérience pertinente de travail, en faisant altemer sessions de cours et stages de formation en entreprise "2).

Aujourd' hui, à l'aube de l'an 2000, alors que connaissances, techniques d'intervention et technologies de pointe sont vite périmées, on peut se demander à juste titre quel type de bagage il reste à l'étudiant après une formation universitaire (ou collégiale)? Selon Nicole Fortin qui a élaboré un document sur la problématique des stages pour le ministère de l'Éducation du Québec (MEQ) en 1984, *ce qui reste de la formation, c'est la capacité d"analyser et de comprendre des situations humaines complexes, cette capacité de s'adapter, de créeretd' inventer sa profession presque quotidiennement. Les stages sont l'espace de formation qui vise l'exercice et l'apprentissage de ces habiletéss ${ }^{(9)}$.
Les stages, peu importe le champd'études de $l^{\prime}$ étudiant, représentent $l^{*}$ une des dimensions importantes de la formation pratique. \&Les stages sont une forme, un element de formation pratique qui s'inscrit dans un ensemble plus vasteque constituent les programmesde formation universitaires(4). Le volet formation pratique peut proposer un large éventail d'activités dirigées, dépendamment des programmes. Il peut s'agir de laboratoires pratiques (ex:: en informatique), de productions théâtrales dirigées ou libres (en art dramatique), de cours-ateliers (URB-1023 Atelier sur les intervenants touristiques en GIT, atelier en joumalisme au baccalauréat en communications), de cours-séminaires sur une thématique précise (URB-4139 Séminaire: réseaux de distribution en tourisme et transports), d'activités sterrains jumelées à un cours (ex.: un voyage en France, organisé dans le cadre du cours URB-4135 = Installations touristiques), etc. Toutes ces activités, qui different des stages, possèdent leurs caractéristiques propres et contribuent à préparer la future vie professionnelle des étudiants.

Bien sûr, l'implication de la gente étudiante dans des activités complémentaires au cheminement académique, aussi appelées activités upara-scolaires», permet également d'enrichir, de complêter les enseignements reçuset d'introduire à sa manière aux réalités de la vie professionnelle en milieux de travail. Nous aborderons d'ailleurs ce point au cours de notre réflexion. Nous ne pouvons nier l'aspect formateur de toutes les activités ci-hautmentionnés, etplus particulièrement, celui des stages en entreprise.

\section{Définition d'un stage}

Stage (cours-stage): activité siglée de formation pratique, inscrite dans un programme d'études qui se réalise dans un organisme $\mathrm{d}^{\prime}$ accueil selon le syllabus prévua à cet effet tet selon un cadre général défini par les modules ${ }^{(5)}$.

Le stage ${ }^{(6)}$ doit être considéré comme un moyen pédagogique, parmi d'autres, pour parfaire la formation pratique et professionnelle. Sa caractéristique principale est de 
mettre à contribution au plan pratique un praticien d'un milieu généralementexterne à $1^{\dagger}$ université.

Ainsi, différenciế des autres activités pratiques d'un programme spécifique, le stage exige de préciser un certain nombre de conditions à respecter, a cause de la collaboration et de l'association d'entreprises externes à la mission de formation de l'université. Tout stage - de niveau universitaire ou collégial - devrait respecter des conditions minimales parmi lesquelles nous retenons ici:

la préparation des étudiants à leur stage;

- l'encadrement et le suivi des ćtudiants avant, pendant et après le stage;

- relevant des modules impliqués, l'évaluation des apprentissages réalisés par lesétudiants durant leurpériode de stage en entreprise;

- la cueillette des réactions des milieux concernes sur les étudiants, la formule et le vécu des stages ainsi que sur les programmes de formation, dont les étudiants deviennent en quelque sorte les ambassadeurs dans leur milieu de stage respectif.

Le baccalauréat en gestion et intervention touristiques (GIT) de l'UQAM nous aidera à comprendre l'importance strategique d'un stage en tourisme pour ensuite confronter les notions et conditions déjà mentionnées avec ce stage en particulier.

\section{La formation en tourisme à I'UQAM}

Le baccalauréat en GIT a pour objectif de former des professionnels du tourisme, capables de gérer le phénomène touristique ot les diverses entreprises qui y sont reliées. Créé en $1978^{\circ}$ pour répondre aux besoins exprimés par les milieux concernés - tant publics que privés - de disposer d'un bassin de gestionnaires spécifiquement formés en tourisme, le baccalaureat intègre des lors au cheminement de ses étudiants un stage en milieu professionnel. Dans le cursus qui présente l'actuel programme et les cours qui le composent, on peut y lire que: *Une familiarisation avec le milieu touristique par l'entremise de travaw pratiques et d'un stage permet aux étudiants une meilleure intégration à ce milieu. $s^{(8)}$

Le baccalauréat en GIT a toujours privilégié une approche multidisciplinaire, propre au phénomène et à l'industrie qui en résulte, pour aborder le tourisme sous ses multiples facettes: sociale, culturelle, environnementale, politique et économique. Dans l'actuel programme, seize cours, suivis par tous les étudiants, forment ce que l'on appelle le tronc commun. Le focus est ici axé sur la compréhension du phénomène touristique, sur les composantes - ou intervenants - de l'industrie et sur l'acquisition d'une solide formation de base en gestion et administration. Par la suite, l'étudiant est appelé a aiguiser sa formation par le choix d'une des trois concentrations offertes. Que l'étudiant décide d'orienter sa formation vers la planification et la creation de produits touristiques, vers la gestion des entreprises touristiques ou vers la gestion et l'administration d'un établissement hôtelier ou de restauration, sa troisieme annee lui apporte sune consolidation des acquis et un approfondissement de la concentration choisie, tout en offrant la possibilite d'appliquer ses connaissances dans le cadre d'un stage ou d'un projet d'intervention ${ }^{(9)}$.

D'emblée, on ressent la décision de faire du stage l'un des éléments-clé du programme, maintenantreconnucommel'undesmeilleurs programmes d'études touristiques au monde par l'Organisation mondiale du Tourisme (OMT). Ce choix de mettre l'emphase sur le stage témoigne d'une volonté modulaire et institutionnelle de partenariat, c'est-â-direun désir réel de collaborer étroitement avec les intervenants des secteurs public et privé de l'industrie touristique québecoise. Collaboration et concertation avec les milieux concernés, afin de répondre adéquatement à leurs besoins, aux aspirations des étudiants ainsi qu'aux nombreux défis auxquels l'industrie estactuellementconfrontée, sont donc au coeur de ce rendez-vous partenarial comme elles doivent être le catalyseur dynamique des préoccupations et des actions de tous les partenaires touristiques.

Cette originale prise de contact *universits/ étudiant/entreprisen devrait être bénéfique à tous les partenaires. En ce qui concerne l'étudiant, elle offre l'opportunité d'un moment d'intégration des acquis académiques dans un milieu concret et peut servir de tremplin, de porte d'entréc a la vie professionnelle. En quatorze annees, la formule de stage au baccalauréat en GIT a connu trois scénarios bien différents.

\section{Historique des stages en GIT}

A la création en 1978, le stage n'ctait pas que terminal. L'étudiant devaiteffectuerun court stage d'une quinzaine de jours, valant trois crédits, à la fin de chacune des trois années de baccalauréat. A l'occasion d'une révision importante du programme au début des années 1980, la formule du cours-stage est modifiée et on opte pour un stage unique et terminal, d'une valeur de six crédits, d'un minimum de trente jours sterrain $*$, le tout réparti en deux blocs de trois crédits chacun, idéalement effectué lors des deux dernières sessions où $l^{\prime}$ étudiant était inscrit ${ }^{(10)}$.

L'année scolaire 1988-1989, à la suite d'une autre révision du programme, voitémerger le troisième et actuel concept de stage au baccalauréat en GIT. La révision profonde d'alors veut mettre davantage l'accent sur l'aspect gestion dans le cheminement académique global et valoriser le stage, en tant qu'activité de synthèse de la formation reçue à l'université. Le module de GIT, son corps professoral et le Service de la formation exteme et des stages (S.F.E.S.) de l'UQAM collaborent étroitement a la concrétisation des stages.

\section{Le stage en tourisme: définition et caractéristiques}

Voici la définition actuellement proposée par les documents d'information a l' intention des stagiaires.

Stage en tourisme: Lestage est l'occasion de manifester des aptitudes complementaires a l'analyse et à l'intervention. Les connaissances acquises sont appliquées au sein d'un organisme, dans un cadre réel de travail professionnel où la recherche et l'action peuvent s'intégrer. Définition d'un mandat de concert avecl'organisme d'accueil. Réalisation de ce mandat au sein de l'organisme, sur le terrain. Rédaction d'un rapport final sous la direction du tuteur universitaire. Présentation de ce rapport dans le cadre de séminaires d'évaluation.

Depuis l'automne 1988, le stage en tourisme se réalise en une seule session, de façon intensive. Il vaut neuf crédits et représente une etape-charnière dans la formation de l'étudiant, prévue dans le demier tiers de son cheminement académique, et précédant de peu la véritable entrée du diplômé sur le marché du travail. Le séjour aterrains doit compter minimalement quarante-cing jours ouvrables. Cette modification constitue un incitatif largement apprécié par les milieux d'accueil, qui peuvent dorénavant bénéficier de l'apport positif d'un étudiant durant une période plus longue et intensive, compara- 
tivement aux précédentes formules de stages.

De plus en plus d'intervenants y voient $l^{\prime}$ occasion de mettre à l'épreuve d'éventuels candidats à des postes rémunérés au sein de leurentreprise. Cette facetten'estpas toujours présente, il faut bien le dire, pour ne pas faire miroiter de faux espoirs aux stagiaires mais ces derniers bénéficieront au moins, s'il y mettent un minimum d'ardeur et de coeur à l'ouvrage, d'une expérience pertinente qui viendra étoffer leur curriculum vitae.

Ilnous faut aussi insister surl'aspect formation que l'onattend des milieux d'accueil. Ceuxci, sur base volontaire, participent d'une grande mission sociale et éducative que les maisons d'enseignement ne peuvent remplir a elles seules, c'est-à-dire préparer une nouvelle génération de praticiens en tourisme pour demain. Ils aident a paufiner, a réajuster et parfois même à valoriser les enseignements reçus dans les colleges et universités. Leur principal bénéfice: un bassin de jeunes professionnels dynamiques que les milieux peuvent former selon leurs propres politiques et standands. De plus, les stagiaires devraient être en mesure d'apporter une contribution éclairée et assez objective découlant d'un environnement externe, a lamission des entreprises qui les accueillent.

On peut déjà voir à ce stade se profiler certains problèmes puisque toutes les situations de stages ne sont pas idylliques comme la vie elle-même. La vie est un long apprentissage; de même, la formation et l'expérience ne sont pas des processus fermés et achevés. A un jumelage étudiant-entreprise en apparencemoins réussi, il est important de faire ressortir des aspects positifs et des observations qui devraient permettre une meilleure adéquation des stages dans l'avenir et aux étudiants, de préciser davantage leur cheminement professionnel.

IIn'estpas obligatoirequ' unstageen tourisme soit rémunéré. D'ailleurs, une majorité des milieux d'accueil potentiels avoue que l'obligation de rémunérer un stagiaire serait un handicap et leur creerait difficulte. Il ne faut pas oublier le contexte coonomique, d'ici mais aussi à l'échelle mondiale, dans lequel évoluent beaucoup de ces milieux que l'on retrouve généralement dans le secteur des petites et moyennes entreprises ( $\mathrm{PME}$ ) ou des organismes privés a but non-lucratif. Les temps sont difficiles pour tous, particulièrement dans le domaine touristique où la concurrence est fétroce. Cela fait aussi partie de la réalité à découvrir pour les stagiaires. II serait inopportun et contraire aux politiques desmaisons d'enseignement, dont l'UQAM, de refuser des lieux et mandats de stages pertinents sur la seule base de la non-rémuneration des stagiaires.

Un stage peut généralement être effectué durant l'un ou l'autre des trimestres scolaires -automne, hiver, printemps/été -, àMontréal, en région, ailleurs au Canada et mẻme à l'étranger. Idéalement, le lieu et le mandat de stage devraient correspondre à la concentration - ou au champ de spécialisation - choisie par l'étudiant. Mais plus important encore est de réaliser que le stage est l'oocasion rêvée de chercher à exploiter les intérêtset les aptitudes développés durant toute la formation, qu'elle soit universitaire ou collégiale. C'est l'opportunité d'aller vérifier sur le *terrain, de comprendre la vie et les rouages d'une entreprise touristique, de se confronter aux stimulantes et difficiles exigences et contraintes de l'industrie touristique des annees 1990.

Il existe une altemative au stage, celle d'un projet d'intervention en tourisme. Celui-ci $s$ adresse a l'etudiant (ou groupe de deux étudiants) qui veut se lancer en affaires, démarrer une petite entreprise: auberge, firme de consultants, agence axée sur l'organisation d'événements ou de réunions d'affaires, etc. Avec le soutiend'organismes et de personnes-ressources spécialisés en ce domaine et sous l'ćtroite supervision d'un tuteur académique, l'étudiant s'initie aux démarches et procédures nécessaires au démarraged' uneentreprise: rédaction deprojet pour recherche de fonds, plan d'affaires, Étude de marché et/ou de faisabilité, etc. L'étudiant n'a pas à s'intégrer, à sćjourner dans un milieuprofessionnelspécifique. Les exigences académiques demeurent néanmoins élevées car le candidat devra faire la preuve de ses capacites de recherche et d'action en vue de l'acceptation du projet d'abord, puis au niveau de sa concrétisation.

Pour le baccalauréat en tourisme cherchant a répondre adéquatement aux besoins de $\mathrm{l}^{\prime}$ industrie, le stage est obligatoire en GIT. II apparaît comme un instrument indispensable puisque le tourisme est une discipline à caractère professionnel bien plus que théorique. Cependant, «Toute pratique est une intelligence des choses. (...) Il est vrai que, pour le théoricien, l'action est au service de la reflexion et que, pour le praticien, la réflexion est au service de l'action. (...), mais tous deux visent une meilleure compréhen- sion des situations humaines et des pratiques sociales, $x^{(11)}$ Formations théorique et pratique se completent. Davantage sensibilisés, les intervenants font maintenant une plus large place à des gens bien formés mais l'expérience est et demeure un atout majeur. Dans bien des entreprises, les jeunes gradués devront débuter au bas de l'échelle avant d'accéder à un poste décisionnel et professionnel. Cette observation renforce la nécessité d'un stage en tourisme qui favorise une première insertion et expérience de travail des étudiants en entreprise.

Cette expérience «terrains vise globalement à répondre aux principaux objectifs suivants:

- L'étudiant aura l'opportunité de se colletailler à la connaissance concrète et au vécu d'un milieu professionnel procis, doncd'undesnombreux secteurs d'activités reliés au tourisme. II pourra ainsi évaluer si ce secteur lui offre des débouchés et des défis professionnels, captivants ou pas.

- Il pourra tenter de mettre en pratique les connaissances acquises durant la formationacadémiquemais aussi, demettre âl'épreuve ses aptitudes à l'observation, à l'analyse et à l'intervention, trois phases qui sont partie intégrante de toute insertion dans un nouveau milieu de travail, quel qu'il soit.

Habitué a la vie d'étudiant, aux travaux scolaires en équipe, il fera l'apprentissage des règles de fonctionnement et des politiques propres a une entreprise touristique ou à un secteur donné. Initiative personnelle, rythme de travail, productivité, travail et relations avec des collegues, respect des directives, etc., autant d'aspects envisagés dans cette expérience unique à caractère hautement individualisé.

- L'étudiant sera en mesure d'évaluer concrètement sa facilité et son habileté à des communications orales et écrites efficaces, par le biais d'entrevues de selection, mais aussi sur le terrains. II aura à cumuler, préparer, verbaliser, rédiger ses observations, ses analyseset régulièrement, des rapports à l'entreprise, en plus d'un rapport final de stage pour l'université ou le college. Sa capacité de synthese, son sens critique et l'obligation de préparer un bilan de l'expérience seront également mis à l'epreuve. 
- Si la formule «projet» est retenue, l'occasion sera donnée de se frotter au milieu des affaires, de mettre à l'épreuve ses capacites $d^{*}$ entrepreneurship et d'innovation ${ }^{(12)}$. L'étudiant réalisera différentes étapes d'un projet de recherche etd' action, en concertation avec quelques organismes professionnels. Evidemment, l'entreprise n'a pas à être opérationnelle au terme du projet.

Globalement, stages et projets se veulent une ouverture aux réalités professionnelles du tourisme afin de faciliter l'intégration au marché du travail, de provoquer un réajustement au niveau de la formation académique, du type d'emploi recherché, et parfois même d'amener l'étudiant à la décision de poursuivre ses études dans une discipline complémentaire. De fait, c'est la confrontation des idéaux avec la réalité concrète d'un milieu de travail.

\section{Lieux de stages et champs d'intervention}

Les organismes et entreprises, intéressés à recevoir des stagiaires formés en tourisme, sont nombreux et variés; ils peuvent répondreàdifférentes attentes des candidats. Dans quels secteurs d'activités les retrouve-t-on et quels sont-ils?

- Différents ministères, aux niveaux tant fédéral que provincial.

- Dessociétés etorganismes para-publics et para-gouvernementaux (ex.: la Régie des Installations Olympiques).

- Des entreprises privées commerciales de services et de distribution de produits touristiques, culturels ou de loisir dans les domaines du transport, de l'hébergement, de la restauration, des attractions; des firmes de consultants.

- Le secteur associatif qui comprend des associations d'intérêt, les Associations touristiques régionales (ATR), les organismes reliés au loisir touristique et au tourisme social, des attractions à but non-lucratif de type muséal de même que les festivalsetévénements majeurs, etc.

Certains individus voudront choisir leur lieu de stage en fonction d"un secteur d'activités précises (compagnies aériennes, organismes gouvernementaux). D'autres entameront leur recherche au niveau des tâches, des fonc- tions, des mandats à réaliser dans une entreprise, peu importe le secteur (ex.: ainsi, on peut travailler au niveau du marketing pour un grossiste en voyages, un Ministère, un événement majeur, un hôtel, etc.). Les tâches a exćcuter à l'intérieur de certains stages apparaîtront parfois assez techniques et opérationnelles; dans d'autres cas, il y a aura davantage d'observation que d'intervention

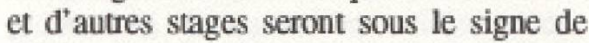
l'analyse et de la recherche. Ne nous le cachons pas: dans le domaine touristique, nous nageons en plein dilemme entre l'exécution - le propre d'un technicien - et la conception - ce qui se rapproche davantage d' une formation universitaire. L'equilibre se retrouve peut-être dans un savant dosage de ces deux pôles. Ce qui est sûr cependant, c'est que pour bien choisir un stage, il faut s'y préparer et y songer longtemps d'avance; l'adage populaire "premiers arrivés, premiers servis» se révélerait assez pertinent quant au sujet qui nous préoccupe.

Les champs d'intervention susceptibles d'intéresser les stagiaires en tourisme sont très diversifiés:

- accueil et information; service a la clientèle;

- administration et gestion d'activités, de personnels, d'equipements;

- collaboration à l'aménagement de sites ou d'équipements récréo-touristiques;

- coordinationd'équipes d'animateursou conception d'un plan d'animation touristique; conception de forfaits et accompagnement de groupes pour des agences réceptives;

- Evvaluation de programmes de formation, de stratégies de marketing ou d'animation;

- aide à l'organisation et à la réalisation de colloques, festivals, congrès, événements majeurs;

- planification de nouveaux produits touristiques, de programmes ou de campagnes de sensibilisation; consultation;

- commercialisation, promotion et communication pour des organismes ou produits touristiques par le biais des départements de ventes, de marketing; conception de plans de communica- tion; travail en publicité, relations de presse, relations publiques et journalisme;

- rechercheet développement par le biais de consultations, sondages, enquêtes, études de marché ou de faisabilité, etc.

Pour profiter pleinement de son expérience de stage et en retirer le maximum de retombées bénéfiques, le futur stagiaire doit considérer l'éventail de possibilités en termes de lieux et de champs d'intervention. Cette dimension de wchoix éclairés nous amène à envisager les trois grandes phases d'uncoursstage: l'avant, le pendant et l'après.

\section{Avant le stage (durée: 6 mois à un an)}

Tout étudiant en tourisme gagne à se préparer et à songer à son stage longtemps à l'avance. Penser au stage est possible des le début d'une formation en tourisme en s'impliquant dans des comités organisateurs d'événements (rallye, petits déjeuners-causerie), en s'initiant aux relations publiques (Comité depromotion), en travaillantàtemps partiel dans le domaine touristique (en hôtellerie, dans des kiosques d'information touristique, dans des agences de voyages au détail ou grossistes, etc.), en tâchant d'être toujours à la fine pointe de l'actualité touristique et de ses dossiers chauds, en cultivant les contacts effectués avec des intervenants touristiques au fil des travaux scolaires, en perfectionnant le français oral et écrit, en approfondissant sa connaissance d'une langue seconde.

Ilestimportantdecemer le secteurd'activités dans lequel on aimerait effectuer un stage. $\AA$ l'UQAM, pour les étudiants en GIT, un inventaire des lieux de stages est disponible pour consultation au S.F.E.S. qui organise régulièrement, en collaboration avec le module de tourisme, des sćances d' information à l'intention des futurs stagiaires. Des ressources humaines, incluant le corps professoral, sont disponibles pour conseiller et guider les étudiants; des ateliers de rédaction de curriculum vitae et de simulation d'entrevues sont également offerts aux gens la session précódant leur stage.

A l'UQAM, toute la coordination administrative de l'opération-stage est assurée par le S.F.E.S.; les démarches personnelles des étudiants sontnaturellement bienvenuesmais l'agent de stage au S.F.E.S. doiten être avisé. Lorsque le processus en vue de l'obtention 
d'un stage est en cours, il n'est pas recommandé de multiplier inutilement les démarches. Le temps est venu de bien définir les objectifs - personnels et professionnels -, de poser des hypothèses que l'on voudra vérifier en cours de stage et de réfléchir à la pratique professionnelle qui sera exercee. Le S.F.E.S. et les tuteurs académiques peuvent être mis à contribution à cet effet. Vient ensuite l'étape de la négociation du mandat de stage puis de son approbationacadémique par un professeur ou chargé de cours. Le tout devrait idéalement être finalisé au moins un mois avant le début du stage dans le milieu d'accueil. Il ne reste alors que le temps pour bien se préparer à son lieu et à ses fonctions, pour démystifier l'endroit et ne pas arriver lă comme un chien dans unjeu dequilles\%. Se documenter, aller sur place avant le début du stage pour fins d'observation, s'assurer que les gens concernés sont aviš́s de l'arrivée d'un stagiaire, préciser que l'on aimerait bénéficier d'une tournée de familiarisation de l'entreprise à l'arrivée, appeler dans les jours précédant le stage pour vérifier qu'un plan de travail sera prépare: voilà quelques moyens pour bien démarrer un stage et signifier aux milieux d'accueil son intérêt et son enthousiasme.

Globalement, l'étudiant doit doncêtrepréparé à observer, à bcouter, à agir et à produire quelque chose qui deviendra la marque de son passage dans le milieu d'accueil; l'attitude positive est aussi de rigueur pour faciliter l'intégration au sclimats de travail de l'entreprise. Il est à noter que si l'on veut effectuer son stage en région ou à l'extérieur du Québec, la démarche globale sera plus longue, pouvant s'échelonner de huit mois à un an.

\section{Pendant le stage}

Ce qui fait la force d'un stage, c'est son caractère intensif; ce qui crée son dynamisme réside dans le degré d' implication, de motivation et d' intérêt, dans le goût, la curiosité et la soif d'apprendre. L'étudiant qui travaille à temps plein ou partiel, inscrit à d"autres cours en plus de vaquer à ses obligations sociales, risque de négliger un peu son stage. Dans le milieu d'accueil, le stagiaire sera appeléà respecter despolitiqueset une confidentialité vis-ă-vis de l'entreprise; cela fait partie de l'ethique professionnelle. L'étudiant se doit d'en être soucieux puisque des futurs stagiaires aimeraient peut-être choisir le même lieu; une expérience négative pourrait mểne s'avérer positive pour un autre individu. Les étudiants deviennent les

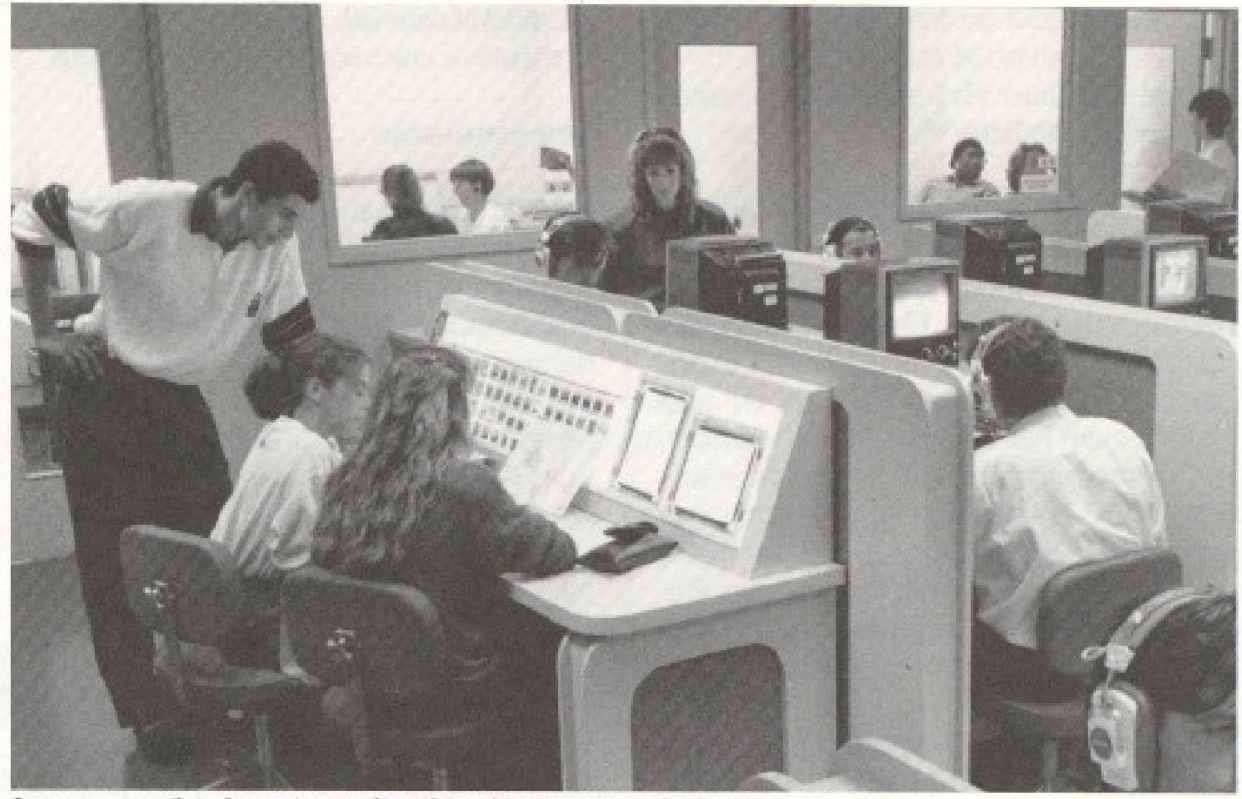

Le rapport trawal-6cole peut etre andione de nambreuses facans, dIT.T. H. Q, certains appreatissages au nivear de stage se fant ser place.

ambassadeurs de leur formation et de leur maison d'enseignement dans les milieux de stages.

Du côté académique, l'encadrement exige souplesse et disponibilité de la part des enseignants. Le tuteur doit s"assurer de suivre le cheminement de l'étudiant par des rencontres individuelles, soutenir au besoin les milieux d'accueil, y rencontrer les superviseurs, voir les étudiants à l' oeuvre et, s'il y a lieu, faciliter la résolution des conflits et des problèmes. De son côté, le stagiaire présente, âson tuteur et au groupe-témoin de stagiaires, un rapport d'étape sur le déroulement du stage ainsi qu'un rapport final. Le contenu de ce demier aura été discuté à l'avance et le sujet, convenu avec le milieu d'accueil. Figurant au menu de la dynamique des stages, quelques rencontres de groupes représentent le lieu d'échange et de partage des expériences sterrains. Il s'agit d'un groupe-support ou témoin, auquel on fait partager les aspects tant positifs que négatifs des réalités vécues. C'est le lieu pour discuter entre futurs professionnels des dissonances entre l'apprentissage académique et la réalité du marché du travail.

\section{Après le stage}

L'étudiant rédige un rapport final, incluant le bilan global de son expérience. Parallèlement à cet exercice de synthèse, il discute de son Évaluation avec le superviseur du milieu d'accueil. L'évaluation du stagiaire par le responsable académique repose donc sur la qualité des apprentissages réalisés, le rapport final et l'évaluation du milieu. Globalement, les stagiaires, le corps professoral et le S.F.E.S., dans le cas de l'UQAM, gagnent à recueillir les réactions des organismes qui ont reçu des étudiants, pour maintenir de fructueuses relations de collaboration avec ces milieux.

\section{Le stage en tourisme: points de vue}

\section{Les milieux d'accueil}

En général satisfaits des stagiaires qu'ils accueillent, les milieux déplorent ne pas être eux-mêmes assez encadrés et soutenus.

Puisqu'ils s'engagent à former des stagiaires, bon nombre de superviseurs apprécieraient être mieux informés et outillés pour remplir adéquatement leur "mission\%. Un consensus se dégage, à savoir une volonté de mieux connaître les types de stages privilégiés par les maisons d'enseignement.

Différentes raisons motivent les entreprises à accueillir des stagiaires; il peut s"agir:

- d'une ressource manquante dans l'organisme;

- de faire une expérimentation avec un nouveau type de professionnel;

- de combler pour une période limitée un poste de professionnel;

- de faire la sélection pour une banque de futurs professionnels;

- de répondre à un besoin réel de service à la communauté; 
- de maintenir un contact plus immédiat avec l'université ou le collège;

- de contribuer à la formation professionnelle d'étudiants ${ }^{(13)}$.

Peu importe la raison qui influence d'ailleurs le déroulement du stage, les milieux souhaitent une relation qui se rapproche de l'échange de services. Fidèles au crédo des années 1980-1990, ils recherchent l'excellence. Cela se traduit de plus en plus fréquemment par un processus de sélection et d'entrevues auprès des candidats potentiels, placés en situation de concurrence et provenant parfois de différentsétablissements scolaires. On veut choisir les meilleurs étudiants, même lorsqu'on ne les rémunc̀re pas; on les veut à la fois autonomes, débrouillards, faisant preuve d'un sens aigu du professionnalisme et respectueux de l'éthique propre au monde du travail. Les entreprises, si elles investissent temps et énergies dans la formation, ne veulent pas perdre au change.

Lesprincipaux points sur lesquels achoppent les milieux de stages vis-à-vis des étudiants sont:

un manque de préparation face à un travail rigoureux et discipline;

- la maîtrise et la qualité souvent insuffisantes de la langue française, orale et 6́crite; l'apprentissage déficient d'une langue seconde (lire l'anglais);

- les objectifs des stagiaires semblent definis trop tardivement; leurs attentes peuvent être démesurées considérant la durce brève d'un stage; les étudiants sont trop idéalistes, ou non-préparés à la réalité des milieux;

- les stages d'intervention, même limités, sont préfétés a ceux strictement centrés sur l'observation.

D'autre part, du côté académique, il faut s'assurer que les organismes sont conscients du temps à consacrer à la supervision et à $1^{*}$ encadrement des étudiants puiqu'il s'agit de stages de formation. Si cela n'est pas possible et réaliste, par exemple durant la haute saison touristique fertile en activités mais parfois peu propice à un encadrement rigoureux et attentif dans certains secteurs, l'organisme devrait refuser d'accueillir un stagiaire. De fait, le secret d'un stage réussi réside dans la disponibilité des parties im- pliquées et dans une communication franche et régulière entre superviseur et stagiaire.

\section{Les étudiants}

Pour la grande majorité des étudiants, le moment du stage en est un de première importance, mais on sous-estime encore trop la définition précise des objectifs à atteindre durant cette expérience. Certains l'appréhendent avec stress, vivent de l'anxiété par peur de l'inconnu ou d'une nouvelle réalité. kes objectifs sont definis, quelques activités sont planifiees et les conditions dans lesquelles il se déroule sont précisées mais il reste toujours une très grande part d' imprévu et d'imprévisibles ${ }^{(14)}$. D'autres trouvent difficile le passage de la théorie à la pratique. Le «terrain» est une réalité plus complexe qư il n'y parâ̂t à première vue et le simple fait de se retrouver dans un milieu professionnel ne permet pas nécessairement de vérifier ou de faire un lien avec les enseignements reçus. D'autres vivent la difficulté de choisir, de prendre une décision qui va orienter leur futur professionnel.

Quant au stage lui-même, tous conviennent de l'importance de l'encadrement et du suivi par la personne-ressource ${ }^{(15)}$ dans le milieu. La première journée est terriblement importante. Si rien de concret n'a êté prévu et que la situation perdure, l'étudiant perdra sa motivation. Le même résultat se produit dans les cas où le milieu d'accueil exige beaucoup, très ou trop vite. $L$ 'incertitude et les situations extrêmes - trop ou trop peu d'encadrement - se vivent douloureusement en stage. Par ailleurs, la perfection n'est pas de ce monde; alors, tenter de cerner ce qui ne va pas et de le communiquer diplomatiquement aux gens concemés peut souvent aider à rectifier une situation fâcheuse. Une façon d'atteindre l'équilibre ou ce qui peut faire la différence entre un bon et un mauvais stage, c'est de savoir exactement, dès le début du stage, les attentes de part et d'autre. Lesexpérienoes vécues en stage façonnent le degré et la qualité de l'intégration et de l'évolution de l'étudiant dans son milieu $d^{7}$ acoueil.

Le stage complété, la formation académique achevée, les diplômés constatent généralement que le stage représente un splus» dans leur apprentissage, en ayant permis un contact direct avec des intervenants. On aura su souvent développer le sens de l'objectivité, seconfronterà laréalité du marché du travail, mettre en application certaines connaissances acquises. La perception est changée: on voit le monde du tourisme moins idéalement et plus objectivement. On réalise que le tourisme est véritablement un secteur d'activites multidisciplinaires et souvent stimulant. On aura saisi les étapes de l'élaboration ou de la réalisation d' un projet. Deux points sombres demeurent au tableau: l'encadrement du milieu parfois «flottants ainsi que la qualité et le niveau peu élevé de certains stages jugés inadéquats, au terme d'une formation collégiale ou universitaire en tourisme.

Un énorme chemin a été parcouru mais il reste encore beaucoup à faire pour valoriser pleinement la formation dans ce secteur d'activités. Les défis sont encore nombreux mais stimulants face à cette problématique des stages. Pour le S.F.E.S. de l'UQAM, porte-parole institutionnel etadministratif en ce qui conceme le lien avec les entreprises, le défi constamment renouvelé est de placer à chaque trimestre tous les candidats stagiaires dans un délai raisonnable et d'effectuer le meilleur jumelage possible lieux-étudiants. Toujours à I'UQAM, le module de GIT continue de raffermir la valorisation du stage mais aussi de la formation globale dispensée et ce, en accentuant ses collaborations avec les partenaires touristiques et son implication dans la vie de l'industrie. Ensemble, tous les partenaires visent l'objectif ultime: whe formation complète, solide et bien ancrée dans la réalité touristique actuelle!

\section{Le mot de la fin}

Au terme de cet exercice, il nous apparaît souhaitable de mettre en lumière quelques éléments importants pour dresser un bilan sommaire de la question. Peu de gens interrogent le rôle fondamental et stratégique du stage en tourisme. Puisqu'on le considere comme partie intégrante de la formation, il faut mettre l'accent auprès des milieux d'accueil sur les notions d'apprentissage et de supervision, incluses dans un processus de formation, c'est-à-dire qu'ils doivent favoriser l'apprentissage des stagiaires et comprendre la nécessité d' une supervision rigoureuse. Ainsi, le stage pourra se définir vraiment comme une expérience supervisce d'apprentissage de la profession dans un milieu identifié comme milieu d'intervention actuel ou potentiel pour des étudiants en tourisme. Il y a donc lieu de réaffirmer la nécessité de former des professionnels capables de réfléchir à leurs interventions, de les concevoir, de les planifier, de les analyser, des professionnels souples, flexibles et capables de se réajuster dans un monde en 
perpétuel changement, des gens capables d'aborder la profession de manière créative et évolutive(16).

Dans leur formation académique, lesétudiants auront été sensibilisés à trois contenus qui entretiennent des rapports étroits entre eux et que l'on retrouve liés au stage: - un environnement socio-economique et culturel, - des institulions, organismes, intervenants, - des activités professionnelles. S'interroger sur les stages permet donc d'aborder la grande question: Mais qu'est-ce qu'apprendre? Un simple accroissement desconnaissances? La capacité de reconstruire à un niveau plus complexe un savoir dejà ellabore? Nous penchons pour la seconde alternative. Le stage peut alors devenir une expérience d'apprentissage unique, dans laquelle l'étudiant sera idealement appele à: se préparer/ observer/comprendre/reproduire/réflechir/ agir/innover. Tous les stages doivent-ils obligatoirement correspondre à toutes ces dimensions? Peut-être pas, mais on peut tendrevers une situation idéale sans toutefois perdre de vue la réalité concrète des entreprises.

Cette opportunité d'aller se confronter au marché du travail - malgré son caractère imparfait - devrait fournir sune premiere expérience professionnelle qui se situe au niveau des exigences de la formation initiale ou de la spécialisation (dépendamment des programmeset des maisons $\mathrm{d}^{+}$enseignement, de niveau collégial ou universitaire ler cycle), sans pour autant devenir ce que l'on appelle de l'expérience professionnelle de travail ${ }^{(17)}$. Il faut encourager les étudiants à viser l'excellence dans leur formation et ce, tant au niveau purement académique que dans leurs implications de vie étudiante; il faut stimuler la soif d'apprendre, le goût d'innover, créer l'environnement propice a la préparation de longue haleine de cetemps fort de la formation que constitue le stage.

Quant aux milieux d"accueil, leur besoin se faitsurtout sentir au niveau de la documentation pour mieux comprendre stages et programmes scolaires et en terme de soutien à l'encadrement. Les milieux sont favorables a un genre de aGuide du superviseur de stages pour réaliser l'ampleur et l'importance accordée aux stages, pour préciser le rôle que l'on attend d'eux. Globalement, $\mathrm{c}^{\dagger}$ est à une plus grande communication que les milieux convient les maisons d'enseignement. Cette démarche devrait leur permettre de mieux accueillir les étudiants, de prévoir sur base régulière des moments d'échange avec eux et surtout de planifier le mandat et le travail confié aux stagiaires. Bien encadrés, motivés et enthousiastes, ces derniers seront aptes à exploiter leur bagage universitaire àl' avantage des entreprises. Ils n'auront plus à se demander ce qu'ils viennent faire dans l'entreprise ou pourquoi on a

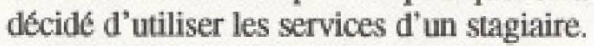
Voilà le défides prochaines années: que tous les partenaires de l'industrie se sensibilisent à une meilleure formation des personnels.

Un personnel qualifié et compétent est l'une des grandes clés du succès pour les entreprises confrontées aux aléas du marché et à la mondialisation des échanges. C'est grâce à la formation que les étudiants reçoivent maintenant que les jeunes professionnels du Québec seront à la hauteur des exigences de demain. Plus les personnels serontcompétents à tous lesniveaux d' intervention, que ce soit dans les transports, l'hébergement ou l'information touristique, l'industrie en entiernes'en portera que mieux. ${ }^{(18)}$

A quel avenir est promise l'industrie touristique et la formation qui s'y rattache? Bien malin qui saurait le dire aujourd' hui mais il y a lieu de continuer à resserrer les liens entre les partenaires, à développer de nouveaux produits touristiques, à faire preuve d'originalité et de créativitéetà susciter des avenues neuves de formation dont témoigne, par exemple, la jeune collaboration UQAMITHQ avec la création d'une concentration en gestion hôtelière et de restauration.

\section{NOTES ET RÉFÉRENCES}

(1) Annick Goulet, Ouand / Universite se rboniente, dans Vie étudiante, janvier/février 1992, p. 10.

(2) Idem.

(3) Nicole Fortin, Penser les atagee, Montreal, ministêre de l'Éducation du Québec (MEO), 1984, p. 27.

(4) UQAM, Politique administrative de la formation externe ot des stages. Vice-rectorat a l'enseignement et à la recherche, juin 1981, p. 2.

(5) Idem, Annexe III, Glossaire, p. 3.

(6) Voir d ce sujet le Rapport du comité concemant la politique púdagogique des stages présenté à la Sous-commission des śtudes de premier cycle et a la Commission des études, juin 1982, pp, 1-2.

(7) L'automne 1978 est important dans l'histoire du tourisme au Oubbec avec le passage du secteur tourisme du MTCP au MICT et la tenue a Sherbrooke du ler Sommet socio-économique sur le tourisme.
(8) Extraits tirés des Depllants sur le baccalauréat en GI.

(9) produits par le module de GIT et l'AEGIT, 1991.

(10) Cette formule sur deux sessions a démotivé plus d'un stagiaire puisque les démarches pouvaient s'etaler sur toute une session et le stage de 30 jours, sur la deuxième. Il apparaitrait que la durée du stage sterrains et l'époque de l'année durant laquelle le stage est effectué peuvent avoir une incidence sur la qualite et I'implication des milieux d'accueil dans l'encadrement des étudiants.

(11) Nicole Fortin, op. clt., p. 19

(12) Un bel exemple de projet qui a vu le jour est RESEAU, centrale de réservations d'hébergement touristique situé a Infotouriste, opéré et fondé par deux diplömés en GIT au moment de leur projet. terminal.

(13) Politique administrative,..., op, elt., p. 13.

(14) Nicole Fortin, op. cit., p. 25. Face d cette peur, cet inconnu des étudiants comparent meme le stage a un kblind datea dont on ne peut prévoir a l'avance le résultat.

(15) Aussi appelée professionnel associé: personne quil assume l'encadrement de l'etudiant dans le milieu de stage: par encadrement, on entend I'insertion du stagiaire dans l'organisme d'accueil, la préparation, la révision et le contróle de ses interventions, I'évaluation des apprentissages faits par le stagiaire. Définition extraite de Politique administrative.... Annexe III. Gloseaire, p. 2.

(16) Voir Nicole Fortin, op. cit., pp. 14 et 24.

(17) Politique administrative,..., op. cit., p. 4.

(18) Extraits du rapport final de stage de Lucie Désilets, étudiante en GIT et stagiaire au secteur Relations avocla presse du ministère du Tourisme. été 1991. 